\title{
Discrete Schwarzschild Models: Constraining Dark Halos and Black Holes
}

\author{
Julio Chanamé ${ }^{1}$, Roeland van der Marel $^{1}$, and Jan Kleyna ${ }^{2}$ \\ ${ }^{1}$ Space Telescope Science Institute, Baltimore, MD 21218, USA \\ email: jchaname@stsci.edu \\ ${ }^{2}$ Institute for Astronomy, University of Hawaii, Honolulu, HI 96822, USA
}

\begin{abstract}
We have developed a code for modeling 3-dimensional kinematical data (i.e., both radial velocity and proper motions) of individual tracers in stellar systems. The code relies on the Schwarzschild method of orbit superposition to find the distribution function (a function of energy, angular momentum, and third integral of motion) that best reproduces all the observations (the positions and velocities of the tracers as well as the overall light distribution) in a given potential. Previous implementations of this method have generally focused on fitting integrated light measurements, instead of data for individual tracers. However, the latter is the only data that are generally available in a large class of problems (e.g., kinematics of tracers in nearby globular clusters, kinematics of planetary nebulae or globular clusters in galaxy halos, etc.). We have performed extensive tests of the fitting process by making use of data generated by a well-tested N-body simulation of an axisymmetric galaxy, quantifying how well the code can recover the known input distribution function and potential.
\end{abstract}

Keywords. galaxies: kinematics and dynamics, galaxies: structure, galaxies: halos, dark matter

To learn about the dark components of galaxies, such as dark halos and black holes, one must study the velocities of tracer objects. Schwarzschild modeling provides one of the most general techniques for interpreting kinematical data. It allows complete freedom in the distribution function (DF) and therefore avoids simplifying assumptions about the velocity isotropy that can lead to spurious conclusions.

Existing Schwarzschild models are generally tailored for continuous line-of-sight velocity distributions, i.e., data from typical long slit spectra of the integrated light from a stellar population. However, new instrumentation in telescopes such as HST, VLT, and Keck are now providing datasets consisting of discrete velocities, as in the cases of globular clusters, planetary nebulae or individual stars at large galactic radii, which constrain dark halo properties, as well as the velocities of individual stars near the centers of globular clusters, which constrain the presence of intermediate-mass black holes.

Also, proper-motion measurements are now becoming available, e.g., for stars in globular clusters. Datasets consisting only of line-of-sight velocities are often insufficient to fully constrain all the complexities of a real stellar system. Proper motions add two additional components of phase-space and allow for much tighter constraints.

The adequate exploitation of these new datasets requires new techniques. In order to use Schwarzschild models with discrete data one must find the maximum of a likelihood function in a few-thousand dimensional space. This is considerably more complicated than the linear matrix equation for the continuous case. We have developed a code that handles this, and extensive tests using simulated data show that we are able to adequately recover the input DF and the mass-to-light ratio (Kleyna, van der Marel, \& Chanamé 2007 , in preparation). Applications to real datasets are in progress. 